\title{
THE FUNDAMENTAL GROUP OF THE DOUBLE OF THE FIGURE-EIGHT KNOT EXTERIOR IS GFERF
}

\author{
D. D. LONG AND A. W. REID
}

\begin{abstract}
We prove that the fundamental group of the double of the figure-eight knot exterior admits a faithful discrete representation into $\mathrm{SO}(4,1 ; \mathbf{R})$, for which the image group is separable on its geometrically finite subgroups.
\end{abstract}

\section{Introduction}

Let $G$ be a group, and let $H$ be a finitely generated subgroup; then $G$ is called $H$-subgroup separable if, given any $g \in G \backslash H$, there exists a subgroup $K<G$ of finite index with $H<K$ and $g \notin K$. The group $G$ is called subgroup separable (or LERF) if $G$ is $H$-subgroup separable for all finitely generated $H<G$.

If $G$ is a discrete group of isometries of hyperbolic $n$-space, then, at present, proving that $G$ is subgroup separable seems to be hard, and it is better to restrict one's attention to the class of geometrically finite subgroups of $G$. If $G$ is $H$-subgroup separable for all geometrically finite subgroups $H$, then $G$ is called GFERF. Thus, for example, since every finitely generated subgroup of a finite covolume Fuchsian group is geometrically finite, by Scott's result [14], Fuchsian groups are GFERF. This property has attracted some attention recently; see, for example, $[\mathbf{1}, \mathbf{6}, \mathbf{1 5}]$.

To describe our main result, we introduce some notation. Let $X$ denote a compact 3-manifold with torus boundary, whose interior admits a complete hyperbolic structure of finite volume which arises as $\mathbf{H}^{3} / \Gamma$, where $\Gamma$ is a subgroup of finite index in some Bianchi group PSL $\left(2, O_{d}\right)$ (see below for an explanation of the notation.) Let the boundary component of $X$ be denoted by $T$, and let $D X=X \cup_{T} X$ be the closed 3-manifold obtained as the double of $X$ over $T$. Our main result, proved in the same spirit as [1], is as follows.

THEOREM 1.1. (i) There are an integer $n \geqslant 4$ and a faithful representation $\rho: G=\pi_{1}(D X) \rightarrow \mathrm{SO}(n, 1 ; \mathbf{R})$ such that $\rho(G)$ is a subgroup of a discrete arithmetic subgroup of $\mathrm{SO}(n, 1 ; \mathbf{R})$. Furthermore, $\rho(G)$ is geometrically finite.

(ii) $\rho(G)$ is GFERF.

This result should be contrasted with the situation for graph manifolds, for which many examples are known not to be LERF (see $[3,7,10]$ ). As is obvious from the existence of an essential torus in $D X, D X$ is not a hyperbolic 3-manifold. The first

Received 15 April 2000; revised 1 August 2000.

2000 Mathematics Subject Classification 20H10.

This work was partially supported by the N.S.F. The second author's work was also partially supported by the Alfred P. Sloan Foundation.

Bull. London Math. Soc. 33 (2001) 391-396 
part of Theorem 1.1 implies that $D X$ is uniformized as a hyperbolic $n$-manifold for some $n \geqslant 4$.

Some notation that we shall employ here is to say that a group $G$ is GFERF if there is a representation $\rho: G \rightarrow \mathrm{SO}(n, 1 ; \mathbf{R})$ such that $\rho(G)$ is GFERF.

As a special case of Theorem 1.1, we note that consideration of $d=3$ gives the following theorem.

THEOREM 1.2. Take $X$ to be the figure-eight knot exterior and let $G=\pi_{1}(D X)$. Then $G$ is GFERF.

We have the following corollary in the language of geometric group theory. Recall that a subgroup $H<G$ is quasi-convex if there is a $K$ such that any geodesic in the Cayley graph of $G$ between two points of $H$ lies within the $K$-neighbourhood of $H$.

COROLlary 1.3. Let $H$ be a quasi-convex subgroup of $G$; then $G$ is $H$-separable.

This follows from work of L. Reeves [12], who established that quasi-convex subgroups of geometrically finite groups of hyperbolic isometries are geometrically finite. A sketch of a proof following the ideas in [4] is given at the end of this paper.

\section{Preliminaries}

We recall some basics about hyperbolic spaces and discrete groups (see [11] for more details).

2.1. Throughout this paper, we shall use the term Kleinian group to mean a discrete subgroup of orientation-preserving isometries of $\mathbf{H}^{n}$. The group of all orientation-preserving isometries of $\mathbf{H}^{n}$ can be identified with $\mathrm{SO}_{0}(n, 1 ; \mathbf{R})$, the connected component of the identity in $\mathrm{SO}(n, 1 ; \mathbf{R})$. We shall sometimes find it convenient to pass between the upper half space model of hyperbolic space and the Lobachevskii space model. In the upper half space model the action of isometries is given by the Poincare extension from $\mathbf{R}^{n-1}$.

Let $\Gamma$ be a finitely generated Kleinian group, and let $C(\Gamma)$ denote the convex core of $\mathbf{H}^{n} / \Gamma$. $\Gamma$ is called geometrically finite if for all $\epsilon>0$, the $\epsilon$-neighbourhood of $C(\Gamma)$, denoted $N_{\epsilon}(C(\Gamma))$, has finite volume.

2.2. We recall the basic combination theorem for amalgams, see [9, Chapter VII]. Let $G$ and $H$ be Kleinian groups acting on $\mathbf{H}^{n}$, and also discontinuously somewhere in the sphere-at-infinity, $S_{\infty}^{n-1}$. Assume that $G \cap H=J$ is non-trivial. An interactive pair for $G$ and $H$ consists of two $J$-invariant non-empty disjoint sets $X$ and $Y$ such that every element of $G \backslash J$ maps $X$ into $Y$, and every element of $H \backslash J$ maps $Y$ into $X$. The interactive pair is called proper if there is a point of $X$ that is not $H$-equivalent to a point of $Y$, or if there a point of $Y$ that is not $G$-equivalent to a point of $X$. We have the following version of the combination theorem.

Theorem 2.1. Let $G, H$ and $J$ be as above, and in addition let them be finitely generated and geometrically finite. Let $(X, Y)$ be a proper interactive pair for $G$ and $H$. Then $\langle G, H\rangle=G *_{J} H$ is a geometrically finite Kleinian group. 
2.3. Let $d$ be a square-free positive integer. We let $O_{d}$ denote the ring of integers in $\mathbf{Q}(\sqrt{-d})$. Then $\operatorname{PSL}\left(2, O_{d}\right)$ is called a Bianchi group, and the collection of these groups determines all the commensurability classes of non-cocompact but finite co-volume arithmetic subgroups of $\operatorname{PSL}(2, \mathbf{C})$; see [5, Chapter 10] and [8], for example.

\section{Proof of Theorem 1.1}

We begin by outlining the idea of the proof. The first stage (following the ideas in [1]) is to embed the group $G$ into an arithmetic subgroup $\Gamma$ (of some $\operatorname{SO}_{0}(n, 1 ; \mathbf{R})$ ) commensurable with an all right reflection group. We then use Theorem 2.1 to show that the double embeds into $\Gamma$, and subsequently appeal to [1, Theorem 3.1].

For ease of exposition, we do this in the case of a figure-eight knot, and then indicate the changes that need to be done in general. Thus, in what follows, $X$ will denote the exterior of the figure-eight knot.

We have the following refinement of some of the analysis in [1]. It will also be convenient to make use of [5, Chapter 10]. See [1] or [5] for more details on quadratic forms and the arithmetic background.

Proposition 3.1. $\quad \mathrm{SO}_{0}(4,1 ; \mathbf{Z})$ is commensurable with a group $\Gamma$ which contains a subgroup $G_{0} \cong \pi_{1}(X)$. Furthermore, $G_{0}$ preserves a co-dimension 1 totally geodesic submanifold of $\mathbf{H}^{4}$.

Proof. Let $p$ be the quaternary form $\langle 1,1,1,-3\rangle$. Notice that this form represents 0 non-trivially, and hence the corresponding arithmetic group $\mathrm{SO}_{0}(p ; \mathbf{Z})$ is noncocompact. This implies that $\mathrm{SO}_{0}(p ; \mathbf{Z})$ is commensurable with some conjugate of an appropriate image of the Bianchi group $\operatorname{PSL}\left(2, O_{3}\right)$.

In fact, as is pointed out in [5, Chapter 10.2, Example 7], $\mathrm{SO}_{0}(p ; \mathbf{Z})$ is the image of the maximal arithmetic Kleinian group $\operatorname{PGL}\left(2, O_{3}\right)$ (see also [8]). In particular,

$$
G_{0}<\operatorname{PSL}\left(2, O_{3}\right)<\operatorname{PGL}\left(2, O_{3}\right) \text {. }
$$

Now the key claim is that $q=\langle 3\rangle \oplus p$ is equivalent over $\mathbf{Q}$ to the form $\langle 1,1,1,1,-1\rangle$; it is visibly equivalent over $\mathbf{R}$. But this follows from the theory of quadratic forms; the version required is stated as [1, Lemma 6.3]. One simply notes that the forms have the same discriminants and Hasse invariants over $\mathbf{Q}$. This gives the required group $\Gamma$ commensurable with $\mathrm{SO}_{0}(4,1 ; \mathbf{Z})$. It is then clear by construction that $G_{0}<\Gamma$ will preserve a co-dimension 1 totally geodesic submanifold of $\mathbf{H}^{4}$.

We also have the following theorem from [1, see Theorem 3.1 and Lemma 3.2].

THEOREM 3.2. The arithmetic group $\mathrm{SO}_{0}(4,1 ; \mathbf{Z})$ is GFERF.

Combining Theorem 3.2 and Proposition 3.1 recovers the fact that $\pi_{1}(X)$ is GFERF.

3.1. Let $\Gamma$ and $G_{0}$ be the groups provided by Proposition 3.1. It is convenient to work in the upper half space model of $\mathbf{H}^{4}$, viewed as the following subspace of $\mathbf{R}^{4}$ with the hyperbolic metric:

$$
\left\{(x, y, z, t) \in \mathbf{R}^{4}: t>0\right\}=\mathbf{R}^{3} \times \mathbf{R}_{+} .
$$


We shall arrange that $G_{0}$ preserves the copy of hyperbolic 3-space determined by the totally geodesic hyperplane $\mathscr{H}$ in $\mathbf{H}^{4}$ given by $\{z=0\}$.

Consider now the action of $G_{0}$ on $S_{\infty}^{3}$. The hyperplane $\mathscr{H}$ meets the sphereat-infinity in the $(x, y)$-plane in $\mathbf{R}^{3}$, and $G_{0}$ preserves the 'upper' and 'lower' half 3 -spaces bounded by this 2-plane. We label this 2-plane by $\mathscr{H}_{0}$. Since the action on $\mathbf{H}^{4}$ is just the Poincare extension of the action on $\mathbf{R}^{3}$, we see that $G_{0}$, acting on these half-spaces, is simply the action by Möbius transformations. In particular, we deduce from this that we can arrange the action of $G_{0}$ on these half-spaces to be that given by the standard representation of $\pi_{1}(X)$ as a subgroup of $\operatorname{PSL}\left(2, O_{3}\right)$; that is, that generated by

$$
\left(\begin{array}{ll}
1 & 1 \\
0 & 1
\end{array}\right) \text { and }\left(\begin{array}{ll}
1 & 0 \\
\omega & 1
\end{array}\right) \text {, }
$$

where $\omega^{2}+\omega+1=0$. Let $H$ denote the peripheral subgroup of $G_{0}$ fixing infinity, which consists of parabolic isometries of $\mathbf{H}^{4}$. Note these have a (unique) common fixed point in $S_{\infty}^{3}$, namely $\infty$.

Now $\mathbf{H}^{4} / \Gamma$ has an end corresponding to the $\Gamma$-orbit of $\infty$, and this end is diffeomorphic to $E \times[0, \infty)$, where $E$ is a closed three-dimensional Euclidean orbifold. Since $G_{0}$ has finite volume acting on $\mathbf{H}^{3}, G_{0}$ is a geometrically finite subgroup of $\Gamma$, and so $\Gamma$ is $G_{0}$-separable, by Theorem 3.2. We may now pass to a finite index subgroup $\Gamma_{1}$ in $\Gamma$, such that the following statements hold:

(i) $X$ is embedded in $\mathbf{H}^{4} / \Gamma_{1}$;

(ii) the Euclidean orbifold $E$, as above, is the 3-torus.

To see this, we argue as follows. Statement (i) is a standard application of separability properties in topology (see [14]). The second statement follows, since the set of elliptic elements in $\Gamma$ fixing $\infty$ is a finite group, and hence we can separate each of these elliptic elements from $G_{0}$. By another application of $G_{0}$-separability, we can pass to a further subgroup of finite index such that $E$ is the 3-torus. It may still be the case that $\Gamma_{1}$ has elements of finite order.

We now uniformize $D X$, as claimed in Theorem 1.1, by a subgroup of infinite index in $\Gamma_{1}$. This will complete the proof of GFERF. For if $K$ is a geometrically finite subgroup of $G$, it is geometrically finite as a subgroup of $\Gamma_{1}$. Now $\Gamma_{1}$ is GFERF; hence $K$ is separable in $\Gamma_{1}$, and therefore in $G$. For if $\gamma \in G \backslash K$, there is a subgroup $\Gamma_{2}$ of finite index in $\Gamma_{1}$ such that $K<\Gamma_{2}$, but $\gamma \notin \Gamma_{2}$. Then $\Gamma_{2} \cap G$ contains $K$ but not $\gamma$.

To uniformize $D X$, we use Theorem 2.1. $X$ is embedded in $\mathbf{H}^{4} / \Gamma_{1}$, and so the boundary torus $T$ of $X$ is embedded in $E$ (recall the notation above), which is just the 3-torus. Thus, since $H$ preserves $\mathscr{H}_{0}$, we can find a parabolic element $p \in \Gamma_{1}$ fixing $\infty$, such that $p$ translates along the $z$-axis by a number much larger than 2 . Thus $\langle H, p\rangle \cong \mathbf{Z}^{3}$ and the said $p$ translates $\mathscr{H}$ far from $\mathscr{H}$ (in the Euclidean metric on $\mathbf{R}^{4}$ ). In particular, this ensures that $G_{0} \cap p G_{0} p^{-1}=H$.

Let $Z=\left\{(x, y, z) \in \mathbf{R}^{3}: z>1\right\}$ and let $Y=\left\{(x, y, z) \in \mathbf{R}^{3}: z<1\right\}$. Note that, since $H$ is acting by translations on the upper and lower half 3 -spaces described above, $H$ preserves $Z$ and $Y$. Furthermore, note that $\mathscr{H}_{0} \subset Y$. We claim that $(Z, Y)$ is a proper interactive pair for $G_{0}$ and $p G_{0} p^{-1}$. Theorem 2.1 completes the proof. 
Recall that in terms of the PSL $\left(2, O_{3}\right)$ action on $\mathbf{H}^{3}$, it follows from the ShimizuLeutbecher (or Jørgenson) inequality that, since any element

$$
\left(\begin{array}{ll}
a & b \\
c & d
\end{array}\right) \in \operatorname{PSL}\left(2, O_{3}\right)
$$

not fixing infinity has $|c| \geqslant 1$, we deduce that $G_{0}$ maps $Z$ into $Y$. Now consider $q \in Y$. The element $p^{-1}(q)$ is translated parallel to the $z$-axis far from $q$ : in particular, $p$ was chosen to translate by more than 2 , so the z-coordinate of $p^{-1}(q)$ is less than -1 . Applying $G_{0}$ either translates parallel to $\mathscr{H}_{0}$, or (as above) the image has $z$-coordinate at least -1 . In any event, we see that applying $p$ then translates into $Z$. Thus $q$ is mapped into $Z$ by $p G_{0} p^{-1}$. Thus $(Z, Y)$ is interactive. To see that it is proper, we argue as follows. As noted above, $\mathscr{H}_{0} \subset Y$. Also, $G_{0}$ preserves $\mathscr{H}_{0}$ : it is essentially the limit set for the action on $\mathbf{H}^{3}$. Such points therefore cannot be $G_{0}$-equivalent to points in $Z$. Hence properness is established.

COROLlary 3.3. Let $M$ be commensurable with the figure-eight knot exterior. Then $\pi_{1}(D M)$ is GFERF.

Proof. We claim that $D M$ is commensurable with $D X$, from which the corollary will follow. $M$ is commensurable with $X$, so there is a finite sheeted covering $N$ of both $X$ and $M$. Note that the torus $T$ is covered by some collection of boundary tori in $N$, and that these cover boundary tori in $M$. By elementary covering space considerations, we see that doubling over $T$ and the respective collections of tori in $M$ and $N$ is compatible with the covering maps on the two copies of $X, N$ and $M$ forming the double. Thus $D M$ is commensurable with $D X$.

3.2. We make some comments on the changes needed to handle the statement of Theorem 1.1. In [1], representations of the Bianchi groups as groups of isometries of six-dimensional hyperbolic space was used to prove GFERF. The passage to six, rather than four as used above, was to handle those $\operatorname{PSL}\left(2, O_{d}\right)$ for which $d$ could not be written as a sum of three squares - every integer is a sum of four squares. Thus, for those $d$ which are the sum of three squares, the argument given for the figure-eight knot applies verbatim. However, for $d$ not a sum of three squares, we must work in hyperbolic 6-space. The argument is then modified accordingly. Corollary 3.3 then also holds in the general setting described.

3.3. The doubling can also be seen geometrically using the ideas in [4]; see also the proof of subcase $\mathrm{C}$ in the proof of [1, Theorem 3.1]. The proof of Corollary 1.3 can be seen in this setting as follows. The group $G$ has a convex hull inside $\mathbf{H}^{4}$ (for example, by taking the convex hull of the limit set), and by taking a point in this hull, we can get an embedding of the Cayley graph of $G$ into $\mathbf{H}^{4}$. Recall that the embedding of the Cayley graph of $G$ into $\mathbf{H}^{4}$ is not quasi-isometric because of the presence of parabolic elements; however, if we remove very small embedded horoballs, and equip the resulting space with the path metric, then it does become a quasi-isometric embedding; such a space is often called a neutered hyperbolic space.

Lemma 3.4. A quasi-convex subgroup $H$ of $\rho(G)$ is geometrically finite.

Proof. Since $G$ is quasi-isometrically embedded into the neutered space, and $H$ is quasi-convex in $G$, we deduce that there is an $L$ with the property that the 
geodesic in the neutered space between any two points in the Cayley graph of $H$ (considered as lying in $\mathbf{H}^{4}$ ) lies within a distance $L$ of the Cayley graph of $H$. If we add the very small horoballs back in, we obtain an $H$-equivariant subset $P$ of $\mathbf{H}^{4}$. The set $P$ is not quite convex, but the actual convex hull $Q$ lies within, say, $10 p$ of $P$, where $p$ is the thin triangles constant for $\mathbf{H}^{4}$.

Apart from a very thin part, $Q$ lies within $L+10 p$ of the Cayley graph of $H$, so that the neutered version $Q^{*} / H$ is compact. It follows that $Q / H$ itself has finite volume, and since a quasi-convex subgroup is finitely generated [2, p. 460], $H$ is geometrically finite, as required.

AcKnOwledgements. The second author would like to thank Emily Hamilton for some useful conversations on subgroup separability, which motivated this paper.

\section{References}

1. I. Agol, D. D. Long and A. W. ReID, 'The Bianchi groups are separable on geometrically finite subgroups', Ann. of Math., to appear.

2. M. R. BRIDSON and A. HAEFliger, Metric spaces of non-positive curvature, Grundlehren Math. Wiss. 319 (Springer, Berlin, 1999).

3. R. G. Burns, A. Karrass and D. Solitar, 'A note on groups with separable finitely generated subgroups', Bull. Austral. Math. Soc. 36 (1987) 153-160.

4. D. COOPER and D. D. LONG, 'Some surface subgroups survive surgery', Preprint, http://www.math.ucsb.edu/ cooper/sssss.ps.

5. J. Elstrodt, F. Grunewald and J. Mennicke, Discontinuous groups and harmonic analysis on three-dimensional hyperbolic spaces (Springer, 1999).

6. R. GiтIK, 'Doubles of groups and hyperbolic LERF 3-manifolds', Ann. Math. 150 (1999) 775-806.

7. D. D. Long and G. Niblo, 'Subgroup separability and 3-manifold groups', Math. Z. 207 (1991) $209-215$.

8. C. Maclachlan and A. W. Reid, 'The arithmetic structure of tetrahedral groups of hyperbolic isometries', Mathematika 36 (1989) 221-240.

9. B. Maskit, Kleinian groups, Grundlehren Math. Wiss. 287 (Springer, Berlin, 1988)

10. W. D. Neumann, 'Immersed and virtually embedded $\pi_{1}$-injective surfaces in graph manifolds', Preprint.

11. J. G. Ratcliffe, Foundations of hyperbolic manifolds, Grad. Texts in Math. 149 (Springer, New York, 1994).

12. L. ReEves, 'Rational subgroups of geometrically finite hyperbolic groups', J. Pure Appl. Algebra, to appear.

13. J. H. RuBINSTEIN and S. WANG, ' $\pi_{1}$-injective surfaces in graph manifolds', Comment. Math. Helv. 73 (1998) 499-515.

14. G. P. ScotT, 'Subgroups of surface groups are almost geometric', J. London Math. Soc. 17 (1978) 555-565; see also correction: ibid. 32 (1985) 217-220.

15. D. WISE, 'Subgroup separability of the figure 8 knot group', Preprint.

Department of Mathematics

University of California

Santa Barbara, CA 93106

USA

long@math.ucsb.edu
Department of Mathematics

University of Texas

Austin, TX 78712

USA

areid@math.utexas.edu 